BNL-112615-2016-JA

\title{
Scalable shear-exfoliation of high-quality phosphorene nanoflakes with reliable electrochemical cycleability in nano batteries
}

\author{
Feng Xu, Binghui Ge, Jing Chen, \\ Linhuo Xin, Hongyu Ma, Chongyang Zhu, Weiwei Xia, \\ Huihua Min, Zhengrui Li, Shengli Li, Kaihao Yu, \\ Lijun Wu, Yiping Cui, Litao Sun, and Yimei Zhu
}

Submitted to 2D Materials

June 2016

Condensed Matter Physics and Material Science Department

Brookhaven National Laboratory

\author{
U.S. Department of Energy \\ USDOE Office of Science (SC), \\ Basic Energy Sciences (BES) (SC-22)
}

\footnotetext{
Notice: This manuscript has been authored by employees of Brookhaven Science Associates, LLC under Contract No. DE-SC0012704 with the U.S. Department of Energy. The publisher by accepting the manuscript for publication acknowledges that the United States Government retains a non-exclusive, paid-up, irrevocable, world-wide license to publish or reproduce the published form of this manuscript, or allow others to do so, for United States Government purposes.
} 


\section{DISCLAIMER}

This report was prepared as an account of work sponsored by an agency of the United States Government. Neither the United States Government nor any agency thereof, nor any of their employees, nor any of their contractors, subcontractors, or their employees, makes any warranty, express or implied, or assumes any legal liability or responsibility for the accuracy, completeness, or any third party's use or the results of such use of any information, apparatus, product, or process disclosed, or represents that its use would not infringe privately owned rights. Reference herein to any specific commercial product, process, or service by trade name, trademark, manufacturer, or otherwise, does not necessarily constitute or imply its endorsement, recommendation, or favoring by the United States Government or any agency thereof or its contractors or subcontractors. The views and opinions of authors expressed herein do not necessarily state or reflect those of the United States Government or any agency thereof. 


\title{
Scalable shear-exfoliation of high-quality phosphorene nanoflakes with reliable electrochemical cycleability in nano batteries
}

\author{
Feng $\mathrm{Xu}^{1,2, *}$, Binghui $\mathrm{Ge}^{3, *}$, Jing Chen ${ }^{4, *}$, Linhuo Xin², Hongyu $\mathrm{Ma}^{5}$, Chongyang $\mathrm{Zhu}^{1}$, Weiwei $\mathrm{Xia}^{1}$, Huihua Min ${ }^{1}$, \\ Zhengrui $\mathrm{Li}^{1}$, Shengli $\mathrm{Li}^{1}$, Kaihao Yu${ }^{1}$, Lijun $\mathrm{Wu}^{2}$, Yiping Cui ${ }^{4}$, Litao Sun ${ }^{1}$ and Yimei Zhu ${ }^{2}$ \\ ${ }^{1}$ SEU-FEI Nano-Pico Center, Key Laboratory of MEMS of the Ministry of Education, Southeast University, Nanjing 210096, China. \\ ${ }^{2}$ Condensed Matter Physics \& Materials Science Department, Brookhaven National Laboratory, Upton, New York 11973, USA. \\ ${ }^{3}$ Institute of Physics, Chinese Academy of Sciences, Beijing 100190, China. ${ }^{4}$ School of Electronic Science and Engineering, Nanjing \\ 210096, China. ${ }^{5}$ Research Center for Internet of Things, China University of Mining and Technology, Xuzhou 221008, China. \\ *These authors contribute equally to this work. Correspondence and requests for materials should be addressed to F.X. (email: \\ fxu@seu.edu.cn) or L.S. (email: slt@seu.edu.cn) or Y.Z. (zhu@bnl.gov).
}

Discovery of atomically thin black phosphorus (called phosphorene) holds promise to be used as an alternative two-dimensional material to graphene and transition metal dichalcogenides especially as an anode material for lithium-ion batteries (LIBs). However, at present bulk black phosphorus (BP) still suffers from rapid capacity fading that results in poor rechargeable performance. Here, for the first time, we use in situ transmission electron microscopy (TEM) to construct nanoscale phosphorene LIBs and visualize the capacity fading mechanism in thick multilayer phosphorene by real time capturing delithiation-induced structural decomposition that reduces electrical conductivity and thus causes irreversibility of lithiated $\mathrm{Li}_{3} \mathrm{P}$ phase. We further demonstrate that few-layer phosphorene successfully circumvents the structural decomposition and holds superior structural restorability, even subjected to multi-cycle lithiation/delithiation processes and concomitant huge volume expansion. This finding affords new experimental insights into thickness-dependent lithium diffusion kinetics in phosphorene. Additionally, a scalable liquid-phase shear exfoliation route has been developed to produce high-quality ultrathin (monolayer or few-layer) phosphorene, only by a high-speed shear mixer or even a household kitchen blender with the shear rate threshold of $\sim 1.25 \times 10^{4} \mathrm{~s}^{-1}$, which will pave the way for potential large-scale applications in LIBs once the rechargeable phosphorene nanoscale batteries can be transferred to industrialized enlargement in the future. 


\section{INTRODUCTION}

Atomically thin two-dimensional (2D) nanomaterials such as graphene and transition metal dichalcogenides (TMDs) have shown unexpected potential application as an anode material for rechargeable lithium-ion batteries (LIBs) due to their large surface areas and outstanding electrical properties ${ }^{1-10}$. However, these materials inherently possess moderate specific theoretical capacities, thus there remains a significant demand for other alternative 2D anode materials. Phosphorene, a monolayer or few-layer black phosphorus (BP) atomic layer(s) ${ }^{11-15}$, has emerged as a promising candidate to $2 \mathrm{D}$ materials due to high theoretical capacity ${ }^{16,17}$, high carrier mobility ${ }^{12,18-20}$, and remarkable in-plane anisotropic properties ${ }^{14,21-25}$. Although much effort has been devoted to theoretical studies of phosphorene LIBs including lithium diffusion kinetics and structural stability ${ }^{26-29}$, no experimental works have been reported so far. At present, only the bulk counterpart of phosphorene, BP, has been experimentally studied as an anode material for $\mathrm{LIBs}^{17,30,31}$. But, huge capacity fading in the first discharge/charge cycle resulted in poor rechargeable performance and limited its commercialization ${ }^{30,31}$. The underlying failure mechanism behind the rapid capacity fading still is not understood fully. Therefore, more in-depth works of experimentally excavating the failure mechanism will be greatly appreciated.

Although staying in theoretical studies for phosphorene $\mathrm{LIBs}^{26-29}$, developing scalable methods in advance to produce high-quality phosphorene still shouldn't be overlooked. Structurally similar to graphite, BP atomic layers are stacked together by weak interlayer forces with significant van der Waals character ${ }^{32,33}$, which makes micromechanical exfoliation (Scotch tape delamination) a reliable laboratory-level technique to yield small-scale high-quality phosphorene ${ }^{34-38}$. Large quantities of other 2D nanomaterials such as graphene and TMDs can be produced by chemical $^{39-43}$ or electrochemical ${ }^{44-46}$ exfoliation, but this approach typically introduces defects or phase transformations that degrade electronic properties, thus not suitable for fabricating high-quality phosphorene. Alternatively, liquid-phase exfoliation by virtue of ultrasonication energy has been proved to be a viable option to prepare high-quality, electronic-grade phosphorene without intermediate chemical reactions ${ }^{4-51}$. However, the scalability of the method is limited by the utilization of sonication as an energy source ${ }^{52}$.

In this report, we employed the shear exfoliation technique ${ }^{52-55}$ to produce highly crystalline monolayer or few-layer phosphorene nanoflakes by using shear force to break down the interlayer van der Waals forces in appropriate solvents. As-exfoliated half-finished dispersions of phosphorene nanoflakes were subjected to further strict purification treatment. The structure, chemistry, and dimensionality of the purified phosphorene nanoflakes are characterized through a comprehensive suite of microscopic and spectroscopic analysis including energy-dispersive X-ray spectroscopy (EDS), high-resolution transmission electron microscopy (TEM), high-angle annular dark field scanning transmission electron microscopy (HAADF-STEM), atomic force microscopy (AFM), scanning electron microscopy (SEM), Raman spectroscopy, and electron energy-loss spectroscopy (EELS). By all of these metrics, the shear-exfoliated 2D phosphorene nanoflakes exhibit competitive properties to mechanically exfoliated counterparts ${ }^{12,34}$. To the best of our knowledge this is the first report of producing phosphorene nanoflakes by shear exfoliation. Importantly, we use in situ transmission electron microscopy (TEM) to construct nanoscale phosphorene LIBs and visualize the aforementioned capacity fading mechanism of thick multilayer phosphorene anodes by real time capturing delithiation-induced structural decomposition that reduces electrical conductivity and thus causes irreversibility of lithiated $\mathrm{Li}_{3} \mathrm{P}$ phase within the phosphorene matrix. So far there are still no published reports on the lithiation/delithiation behaviors of phosphorene nanoflakes by in situ TEM. We further demonstrate that few-layer phosphorene successfully circumvents the structural decomposition and holds superior structural restorability, even subjected to multi-cycle lithiation/delithiation processes and concomitant huge volume expansion. This finding affords new experimental insights into thickness-dependent lithium diffusion kinetics in phosphorene. Overall, our scalable production method of high-quality phosphorene and fundamental understanding of phosphorene lithiation/delithiation behaviors have make the pave for potential large-scale applications of rechargeable phosphorene LIBs.

\section{RESULTS AND DISCUDDION}

Bulk BP crystals are exfoliated in organic solvents that are detailed in the Methods section. The whole exfoliation procedure is carried out in a glovebox with argon gas using a mixing head where a 4-blade rotor sits within a fixed screen known as the stator, as illustrated in Figure 1a. Typical features of the mixing head are narrow gap $\left(d_{\text {gap }}=0.2\right.$ $\mathrm{mm}$ ) between the rotor and stator, and high rotor speed $(N)$ creating a high shear rate $(\gamma)$ within the gap. Figure $1 \mathrm{~b}$ show a schematic of BP shear-exfoliation process in the laminar flow regimes that typically occur within the gaps. In a representative experiment, a handful of ground BP powder $(0.05 \mathrm{mg} / \mathrm{mL})$ was immersed into $N$-methyl-pyrrolidone (NMP) in a conical tube (Figure 1a). After the shear exfoliation with predetermined time and rotation speed, turbid dispersion (Figure 1b) was obtained that was further purified by centrifugation to remove larger unexfoliated BP crystals. The resulting stable phosphorene dispersions display a brown to pale yellow color as a function of centrifugal rotation speed, as seen in Figure 1c. Additionally, turbulence-assisted shear rate from a household kitchen blender also can be utilized to exfoliate the bulk BP (This section will be expatiated hereinafter). 
Following the shear exfoliation and centrifugation in NMP, the phosphorene dispersions were further filtrated by polytetrafluoroethylene (PTFE) membrane and repeatedly rinsed by isopropyl alcohol (IPA) to remove NMP residual. The stacked phosphorene nanoflakes on PTFE membrane (Figure S1a) were renewedly dispersed in IPA for subsequent microscopic and spectroscopic characterizations. Figure 2a shows a typical HAADF-STEM image of an ultrathin phosphorene nanoflake on a lacy carbon support, revealing its uniformity in thickness. The nanoflake was isolated by centrifugating the as-exfoliated dispersions at a rotation speed of $6000 \mathrm{rpm}$ (see Figure 1c). Its chemical purity is inspected by the EDS analysis. The EDS result (Figure S2) shows that the nanoflake only consist of phosphorus. Comparison between the EDS mapping images (Figure 2b,c) and HAADF-STEM image (Figure 2a) also indicates the high purity of the nanoflake after thoroughly rinsing the shear-exfoliated products, no obvious $\mathrm{C}$ element from residual organic solvent is found on the surface of the nanoflake. Moreover, the chemical quality of the nanoflake is further checked by EELS (Figure 2d,e). The invisible plasmon peak in low-loss EELS (Figure 2d) proves that the nanoflake is so thin that plasmon peak signal can't be detected ${ }^{56}$. The characteristic core-loss peak at $133 \mathrm{eV}$ corresponding to the $\mathrm{P}$ $L_{2,3}$ edge is observed clearly (Figure $\left.2 \mathrm{e}\right)^{48}$. Absence of the signal $\mathrm{P}_{x} \mathrm{O}_{y}$ peak loss confirms the phosphorus elementary substance all over the whole nanoflake.

The schematic showing the atomic structure of BP crystal along side-view and top-view projections is illustrated in Figure 2f. Monolayer phosphorene consists of $\mathrm{P}$ atoms stacked in puckered subplanes, and each $\mathrm{P}$ atom is bonded with two adjacent atoms lying in the same plane and with one $\mathrm{P}$ atom from another different plane ${ }^{26}$. Here, the monolayer characteristic is clearly resolved for the phosphorene nanoflake of Figure 2a by high-resolution TEM (HRTEM) imaging and corresponding SAED pattern along [010] projection, as reflected in Figure 2g,h. No noticeable structure defects are observed in the HRTEM image. A filtered section of the HRTEM image (upper right inset in Figure 2g) shows atomic-level structure characteristic of monolayer phosphorene, which obviously is different from the ABA stacking order of multilayer phosphorene (Figure S3). Moreover, we also used XHREM software to perform TEM simulations (viewing from the (001) direction) on monolayer and multilayer phosphorene structures (Figure S3). The simulated monolayer structure (lower right inset in Figure 2g) perfectly matches the imaged HRTEM structure, further corroborating the successful exfoliation of monolayer phosphorene. These TEM analyses provide strong evidence that high-quality monolayer phosphorene with highly crystalline nature can be produced by shear exfoliation. This conclusion also is corroborated by Raman spectroscopy, as given in Figure S4. Four modes are observed at $363 \mathrm{~cm}^{-1}$, $438 \mathrm{~cm}^{-1}, 465 \mathrm{~cm}^{-1}$, and $523 \mathrm{~cm}^{-1}$ assigned to the $\mathrm{A}_{\mathrm{g}}{ }^{1}, \mathrm{~B}_{2 \mathrm{~g}}$, and $\mathrm{A}_{\mathrm{g}}{ }^{2}$ photon modes for phosphorene nanoflakes ${ }^{15,57-60}$ and the TO phonon mode for the Si substrate, respectively. The Raman spectrum is well in accord with the previous results of phosphorene nanoflakes produced by mechanical exfoliation.

For further AFM analysis of isolated phosphorene nanoflakes, samples were prepared in an Ar glovebox by spin-coating the phosphorene dispersions in IPA onto $200 \mathrm{~nm} \mathrm{SiO}{ }_{2}$-coated silicon substrates and then were stored in Ar gas-filled sealing bags until AFM characterization. In Figure 3a, an individual phosphorene nanoflake is imaged by AFM equipped with an environmental cell with dry, ultrahigh purity $\mathrm{N}_{2}$ gas. A step height of $\sim 0.85$ nm measured at the nanoflake edge confirms the presence of monolayer phosphorene. No obvious bubbles, droplets, or other signs of phosphorene degradation are found. Even though the step height is slightly larger than the theoretical value of $0.52 \mathrm{~nm}$ for monolayer phosphorene, we generally consider that the AFM-measured thickness value of a monolayer 2D nanoflake is larger than the theoretical value due to the roughness height of $\mathrm{SiO}_{2} / \mathrm{Si}$ substrate; this phenomenon is frequently observed in graphene and TMDs' cases $^{61,62}$. In addition, phosphorene nanoflakes isolated by centrifugating at low rotation speeds also were observed by AFM (Figure S5), and step heights of 0.9-3.5 nm indicate coexistence of monolayer and few-layer phosphorene nanoflakes produced by the shear exfoliation. The dimensionality and size of filtrated nanoflakes by PTFE membrane are found to be up to tens of micrometers, as shown in SEM images of Figure 3b-d and Figure S1b-d.

The mechanism of shear-exfoliating graphite has been well expounded by Coleman and colleagues ${ }^{52-55}$. Just as its name implies, the shear rate $(\gamma)$ is critical for exfoliation. The equation of shear rate can be written as: $\gamma \approx \pi \cdot N \cdot\left(2 R_{\mathrm{r}}\right) / d_{\text {gap }}$, where $N, R_{\mathrm{r}}, d_{\text {gap }}$ are rotor speed, rotor radius, and rotor-stator gap, respectively ${ }^{52,63}$. Obviously, the shear rate increases proportionally with the rotor speed provided that the mixing head of rotor-stator is fixed $\left(R_{\mathrm{r}}=16 \mathrm{~mm}, d_{\text {gap }}=0.2 \mathrm{~mm}\right)$. Interestingly, we found from TEM characterizations that large quantities of high-quality phosphorene nanoflakes were produced only above a minimum rotor speed of $\sim 1500 \mathrm{rpm}$. This implies that a minimum shear rate of $\sim 1.25 \times 10^{4} \mathrm{~s}^{-1}$ is a general requirement in our experiments. According to calculation equation of Reynolds-number, $\operatorname{Re}=N \cdot\left(2 R_{\mathrm{r}}\right)^{2} \cdot \rho / \eta$, where $\rho$ and $\eta$ is the volume and viscosity of the solvent NMP, respectively ${ }^{63}$, the minimum shear rate can be obtained within the laminar flow regime ( $\operatorname{Re}<10^{4}$ ), showing turbulence flow ( $\operatorname{Re}>10^{4}$ ) not to be compulsory for shear exfoliation. The exfoliation process in the laminar flow regime is schematically shown in Figure $1 \mathrm{~b}$ by modeling exfoliation as shear stress $(F)$-induced interlayer sliding in NMP. According to the previous reports, the same exfoliation mechanism should take place not only in laminar regime but also in turbulent regime ${ }^{52}$. This means that any high-speed mixing head that generates the shear rate higher than $\sim 1.25 \times 10^{4} \mathrm{~s}^{-1}$ is suitable for BP exfoliation. Here, we further demonstrate that phosphorene nanoflakes also can be successfully produced using a Joyong kitchen blender (Figure S6a,b and Movie S1). Its rotating blades can generate rotation speed of $\sim 15500-22000$ rpm that would result in 
fully developed turbulence $\left(\mathrm{Re}>10^{4}\right)^{64}$. Assuming a rated power input of $250 \mathrm{~W}$ is completely dissipated via turbulence, the shear rate can be calculated from $\gamma \approx(P /(V \cdot \eta))^{0.5}$, where $V$ and $\eta$ is the volume and viscosity of the solvent NMP, respectively ${ }^{65,66}$. When $200 \mathrm{~mL}$ of NMP was used, the $\gamma$ is $2.73 \times 10^{4} \mathrm{~s}^{-1}$ which is larger than the shear rate threshold of $\sim 1.25 \times 10^{4} \mathrm{~s}^{-1}$ for BP delamination. As-exfoliated phosphorene nanoflakes were analyzed by Raman spectroscopy (Figure S6c), TEM imaging (Figure S6d-f), and EELS (Figure S7), and no perceptible differences in flake morphology, purity, and degree of crystallinity are found compare with the aforementioned product prepared by the rotor-stator system. We also note that these nanoflakes are virtually indistinguishable from the sonication-exfoliated counterparts $^{47-51}$ both in terms of size and quality, indicating that well-exfoliated, high-purity, highly crystalline phosphorene nanoflakes can be produced using a broad range of shear exfoliation conditions.

Bulk BP has been experimentally studied as an anode material in $\operatorname{LIBs}^{16,17,30,31}$. But, successful commercialization of BP anodes has been impeded by rapid capacity fading in the first discharge/charge cycle that resulted in poor rechargeable performance. Although both the experimental ${ }^{17}$ and theoretical ${ }^{27}$ studies considered that lithiation-induced large volume expansion and thus loss of electrical contact resulted in the rapid capacity fading, no direct and credible evidences prove that the lithiation process should be responsible for the failure. The underlying failure mechanism behind the rapid capacity fading still is not understood fully. As to phosphorene, much effort still mainly focuses on its theoretical studies as an anode material for $\operatorname{LIBs}^{26-29}$. So far, no experimental works have been conducted to investigate its electrochemical lithiation/delithiation behaviors. Therefore, further works are needed to excavate the failure mechanism of bulk BP anodes and investigate the electrochemical performance of phosphorene anodes. Here, our high-quality shear-exfoliated phosphorene nanoflakes provide the possibility of in situ observing the morphological and structural evolution during their lithiation/delithiation processes via TEM, and of unraveling lithium diffusion and reaction kinetics mechanism at the nanoscale ${ }^{67}$. Figure 4a schematically illustrates the in situ ithiation/delithiation experiment of an individual phosphorene nanoflake via a TEM-STM sample holder (Nanofactory Instruments) with a biasing system (Figure 4b). The nanoflakes were glued to the half copper grid with conductive epoxy and then were touched to a sharp tungsten tip that was associated to a piezo-driven biasing-probe, forming an electrical circuit by applying a bias voltage. A lithium metal attached to a tungsten tip was served as the counter electrode and the naturally grown $\mathrm{Li}_{2} \mathrm{O}$ layer on the surface of $\mathrm{Li}$ metal was used as a solid-state electrolyte to allow the diffusion of $\mathrm{Li}^{+}$ions ${ }^{68}$.

Figure 4c shows the TEM image of the nano-LIB constructed with a slight thick phosphorene nanoflake inside the TEM. The pristine nanoflake is corresponding to the product centrifugated by $1000 \mathrm{rpm}$ rotation speed (see Figure 1c). Its structure can be identified as orthorhombic-phase BP (JCPDS no. 73-1358, space group Cmca (64)), as indicated in its single-crystalline SAED pattern (Figure 4d). A typical lithiation experiment was initiated by applying a constant potential of $-1 \mathrm{~V}$ to the phosphorene nanoflake (as the nano-LIB's anode) with respect to the Li counter electrode to drive the electrons and $\mathrm{Li}^{+}$ions flow across the circuit after the two electrodes were contacted. Figure 4e-g show time-resolved TEM images from video frames (Movie S2) during the electrochemical lithiation process. It's found that the nanoflake longitudinally elongated from $1.7 \mu \mathrm{m}$ to $2.3 \mu \mathrm{m}$ upon $\mathrm{Li}^{+}$intercalation, with an expansion rate of $36 \%$. The nearly same expansion rate of 35\% from $1 \mu \mathrm{m}$ to $1.35 \mu \mathrm{m}$ also was observed for the lateral direction, which implies the same diffusion rate of $\mathrm{Li}^{+}$ions at different directions. The isotropic lithiation behavior isn't consistent with the theoretical studies that reveal the anisotropic lithium diffusion on the surface of monolayer phosphorene. Especially, density functional theory (DFT) calculations by Zhang, et al. concluded that the shallow energy barrier $(0.08 \mathrm{eV})$ of Li diffusion on monolayer phosphorene along zigzag direction leaded to an ultrahigh diffusivity, which was estimated to be $10^{2}\left(10^{4}\right)$ times faster than that on $\mathrm{MoS}_{2}$ (graphene) ${ }^{26}$. On the contrary, the large energy barrier $(0.68 \mathrm{eV})$ along the armchair direction resulted in a nearly forbidden diffusion. The DFT calculations further point out that the huge difference in diffusion energy barrier along the zigzag and armchair directions could remarkably be reduced for multilayer phosphorene nanoflakes, which can well explain the observed isotropic lithiation behavior in the slightly thick phosphorene nanoflake. Monitoring microstructure evolution of the nanoflake affords the opportunity to better understand the lithiation process and phase transition. After lithiation for $20 \mathrm{~s}$, the SAED exhibits discontinuous ring-like diffraction spots superimposed on diffuse amorphous halos (Figure 4h); the diffraction spots can be indexed as hexagonal-phase $\mathrm{Li}_{3} \mathrm{P}$ (JCPDS no. 74-1160, space group P63/mmc (194)). The diffuse amorphous halos can derive from various transitional $\mathrm{Li}_{x} \mathrm{P}$ phases including $\mathrm{LiP}_{7}, \mathrm{LiP}_{5}$, and $\mathrm{Li}_{3} \mathrm{P}_{7}$, although no characteristic diffraction patterns corresponding to any of these phases can be identified. The transitional $\mathrm{Li}_{\mathrm{x}} \mathrm{P}$ phases were typically amorphous and also occurred in bulk BP anodes with the full phase transition from $\mathrm{P}$ to $\mathrm{Li}_{3} \mathrm{P}^{30,31}$.

The electrochemical delithiation behavior also was in situ inspected by applying a reversed constant potential of $1 \mathrm{~V}$, as shown in Figure 4i-k and Movie S3. Interestingly, the expected dimensional shrinkage associated with the deintercalation of $\mathrm{Li}^{+}$ions didn't take place for the nanoflake; instead, the nanoflake suddenly decomposed. The decomposition began from one end contacting lithium counter electrode (Figure $4 \mathrm{j}$ ) and then rapidly propagated to the other end (Figure 4k), forming discrete regions in the nanoflake matrix. A mass of residual lithium in the formation of $\mathrm{Li}_{3} \mathrm{P}$ phase still existed in the delithiated nanoflake, as reflected in the SAED pattern (Figure 4l) and EELS data (Figure S8). The discrete regions in the delithiated nanoflake resulted in loss of electrical conductivity, as proved by the $I$ - $V$ curves before lithiation and after delithiation (Figure S9). As a result, the $\mathrm{Li}_{3} \mathrm{P} \leftrightarrow \mathrm{P}$ reversible conversion was terminated. 
Here, for the first time, we revealed the capacity fading mechanism in bulk BP anodes should be attributed to the delithiation-induced structural decomposition, rather than lithiation-induced volume expansion. This uncommon finding subverts the commonly accepted understanding, namely that the anode decomposition and fracture usually take place only during the lithiation process due to the large volume expansion, as observed in other anode materials such as $\mathrm{ZnO}$, $\mathrm{Fe}_{2} \mathrm{O}_{3}, \mathrm{SnO}_{2}, \mathrm{Si}, \mathrm{Ge}, \mathrm{Co}_{3} \mathrm{O}_{4}, \mathrm{CeO}_{2}, \mathrm{CoS}_{2}$, etc. However, the delithiation-induced structural failure has not been discussed in the previous reports. The uncommon finding will afford us new insight into re-optimizing the viable bulk BP anodes in the future. Additionally, the anisotropic lithiation behavior and delithiation-induced structural decomposition were repeatedly corroborated by additional in situ lithiation/delithiation experiments (Figure S10 and Movie S4).

Fortunately, we have found that few-layer phosphorene nanoflakes can keep structural integrity even subjected to multi-cycle lithiation/delithiation processes (Figure 5 and Movie S5-7). Here we use the centrifugation at $6000 \mathrm{rpm}$ rotation speed to screen out monolayer and few-layer phosphorene nanoflakes (see Figure 1c). After applying a constant potential of $-1 \mathrm{~V}$ to an individual few-layer phosphorene nanoflake with respect to the Li counter electrode, the lithiation resulted in a lateral inflation from $1.9 \mu \mathrm{m}$ to $2.4 \mu \mathrm{m}$ with an expansion rate of $26 \%$ (Figure 5a-c and Movie S5). However, the longitudinal expansion didn't take place, which implies different diffusion rates of $\mathrm{Li}^{+}$ions at different directions (such as zigzag direction and armchair direction). The anisotropic lithiation behavior is well consistent with the DFT theoretical studies concerning the anisotropic lithium diffusion on monolayer phosphorene. According to DFT calculations, the large energy barrier of Li diffusion on monolayer phosphorene along the armchair direction resulted in a nearly forbidden diffusion compared with an ultrahigh Li diffusivity along zigzag direction with the shallow energy barrier. In a way, the theoretical result also can be used to account for the anisotropic lithiation behaviors in the in situ experiment, proving that the exfoliated few-layer phosphorene is ultrathin and nearly comparable to monolayer phosphorene.

Excitingly, the nanoflake could completely restore its original morphology and size (Figure 5d-f and Movie S5) after the delithiation, and we didn't observe any visible structural decomposition that had occurred in the thick phosphorene nanoflakes. Significantly, the nearly same expansion rate and restorability in morphology were kept after the second lithiation/delithiation cycle, as shown in Figure 5g-l and Movie S6. Even after dozens of lithiation/delithiation cycles, the few-layer nanoflake still can restore its original morphology and size provided that the applied potential is fixed. When a higher potential of $-3 \mathrm{~V}$ was used to lithiate the nanoflake, a larger lateral expansion rate of $45 \%$ was obtained (Figure 5m-o and Movie S7). The longitudinal expansion wasn't found. The large expansion yet didn't result in the structural decomposition during the delithiation by applying an equivalent reversed potential (Figure 5p-r and Movie S7). The structural stability also was verified by additional dozens of lithiation/delithiation cycles. This finding provides powerful evidence that the ultrathin phosphorene nanoflakes are more suitable for applications in LIBS due to superior electrochemical cycleability compared with the bulk BP anodes, and thus possess the great commercialization prospect in high-performance LIBs.

\section{METHODS}

\section{Shear Exfoliation and Purification.}

Shear exfoliation of bulk BP crystals can be carried out both in the laminar regime and in the turbulence regime which can be obtained using a B25 laboratory shear mixer (Shanghai BRT Company) and a household kitchen blender (Joyong Company), respectively. The main component of the shear mixer is a mixing head where a 4-blade rotor sits within a fixed screen known as the stator. In all experiments, only the rotor with a radius of 16 mm was used, and thus the gap between the rotor and stator was invariable, $\sim 0.2 \mathrm{~mm}$. In a typical experiment, bulk BP crystals synthesized by the high-temperature/high-pressure method ${ }^{31}$ that was initiated by Bridgeman ${ }^{31}$.

\section{Atomic Force Microscopy}

AFM was performed on drop-cast flakes on $\mathrm{Si} / \mathrm{SiO}_{2}$ substrates using an Icon Bruker system in standard tapping mode. It was tried to minimize the time between sample preparation and AFM experiments in order to reduce the degradation of the flakes.

\section{Scanning Electron Microscopy}

SEM was performed using a Carl-Zeiss electron microscope integrated in a Raith e-LiNE plus electron-beam lithography system. The images were acquired at $20 \mathrm{kV}$ acceleration voltage and with a $30 \mu \mathrm{m}$ aperture size.

\section{Raman Spectroscopy}

The polarization/orientation Raman measurements of the liquid exfoliated flakes were performed using a HORIBA 
XploRA instrument. The excitation wavelength was $532 \mathrm{~nm}$ with $1.0 \mathrm{~mW}$ focused on individual flakes of phosphorene using a $100 \times$ microscope objective. Data were collected without a Raman analyzer.

Samples were mounted on a rotational stage with $15^{\circ}$ rotational gradations. The BP nanoflakes that appeared the most uniform based upon their color when viewed with white reflected light were selected for polarization/orientation Raman analysis. This was done to avoid convolution of spatially varying structural contributions to the spectra as the sample's orientation with respect to the incident laser polarization was varied. Spectra were collected in $15^{\circ}$ rotational increments from $0^{\circ}$ to $180^{\circ}$ with respect to the laboratory frame.

\section{Transmission Electron Microscopy}

The exfoliated flakes were characterized in the aberration-corrected JEOL ARM200CF scanning TEM (STEM)/TEM operated at electron energy of $80 \mathrm{keV}$ to minimize damage caused by the electron beam. The microscope was equipped with a cold field-emission source, which yielded $0.35 \mathrm{eV}$ energy resolution and $1.2 \AA$ spatial resolution with the probe spherical-aberration corrector in STEM mode, and 2 A resolution in TEM mode. A convergence semiangle of 16.5 mrad was used for both STEM imaging and EELS. For HAADF-STEM imaging, a 90 mrad collection inner angle was used. For EELS, a collection semiangle of 71 mrad was used. A digital micrograph (Gatan, Inc.,USA) was utilized for all data acquisition. For sample preparation, the dispersed nanoflakes in IPA were drop-cast on a lacey-carbon TEM grid. The samples were then lamped for $15 \mathrm{~min}$ to dry out the solvent and immediately loaded into the microscope. High-resolution TEM images of both mono- and multilayer BP were simulated using Kirkland multislice code using the following TEM parameters: incident beam energy: $80 \mathrm{keV}$; thermal vibration temperature: $300 \mathrm{~K}$; spherical aberration $C_{\mathrm{s}}: 0.5 \mathrm{~mm}$; defocus for single layer: $30 \AA$; defocus for multilayer: $48 \AA$; objective aperture size: $500 \mathrm{mrad}$.

\section{In Situ Electrical Measurement and Nanoscale Lithium-Ion Battery Construction}


1. Luo, B. \& Zhi, L. J. Design and construction of three dimensional graphene-based composites for lithium ion battery applications. Energy Environ. Sci. 8, 456-477 (2015).

2. Zhang, C. Z. et al. Synthesis of phosphorus-doped graphene and its multifunctional applications for oxygen reduction reaction and lithium ion batteries. Adv. Mater. 25, 49324-937 (2013).

3. Yoo, E. J. et al. Large reversible Li storage of graphene nanosheet families for use in rechargeable lithium ion batteries. Nano Lett. 8, 2277-2282 (2008).

4. $\mathrm{Wu}, \mathrm{Z}$. et al. Doped graphene sheets as anode materials with superhigh rate and large capacity for lithium ion batteries. ACS Nano 5, 5463-5471 (2011).

5. Mukherjee, R. et al. Photothermally reduced graphene as high-power anodes for lithium-ion batteries. ACS Nano 6, 7867-7878 (2012).

6. Xiao, J. et al. Exfoliated $\mathrm{MoS}_{2}$ nanocomposite as an anode material for lithium ion batteries. Chem. Mater. 22, 4522-4524 (2010).

7. Stephenson, T. et al. Lithium ion battery applications of molybdenum disulfide $\left(\mathrm{MoS}_{2}\right)$ nanocomposites. Energy Environ. Sci. 7, 209-231 (2014).

8. Bhandavat, R., David, L. \& Singh, G. Synthesis of surface-functionalized $\mathrm{WS}_{2}$ nanosheets and performance as Li-ion battery anodes. J. Phys. Chem. Lett. 3, 1523-1530 (2012).

9. Liu, Y., Zhu, M. Q. \& Chen, D. Sheet-like MoSe2/C composites with enhanced Li-ion storage properties. J. Mater. Chem. A 3, 11857-11862 (2015).

10. Wang, G. et al. Two-dimensional $\mathrm{SnS}_{2} @ P A N I$ nanoplates with high capacity and excellent stability for lithium-ion batteries. J. Mater. Chem. A 3, 3659-3666 (2015).

11. Reich, E. S. Phosphorene excites materials scientists. Nature 506, 19 (2014).

12. Liu, H. et al. Phosphorene: an unexplored 2D semiconductor with a high hole mobility. ACS Nano 8, $4033-4041$ (2015)

13. Ziletti, A. et al. Oxygen defects in phosphorene. Phys. Rev. Lett. 114, 046801 (2015).

14. Xia, F. N., Wang, H. \& Jia Y. C. Rediscovering black phosphorus as an anisotropic layered material for optoelectronics and electronics. Nat. Commun. 5, 4458 (2014).

15. Lu, W. L. et al. Plasma-assisted fabrication of monolayer phosphorene and its raman characterization. Nano Res. 7, 853-859 (2014).

16. Stan, M. C. et al. Puzzling out the origin of the electrochemical activity of black $\mathrm{P}$ as a negative electrode material for lithium-ion batteries. J. Mater. Chem. A 1, 5293-5300 (2013).

17. Sun, J. et al. Formation of stable phosphorus-carbon bond for enhanced performance in black phosphorus nanoparticle-graphite composite battery anodes. Nano Lett. 14, 4573-4580 (2014).

18. Li, L. K. et al. Black phosphorus field-effect transistors. Nat. Mater. 9, 372-377 (2014).

19. Buscema, M. et al. Fast and broadband photoresponse of few-layer black phosphorus field-effect transistors. Nano Lett. 14, 3347-3352 (2014).

20. Yang, Z. B. et al. Field-effect transistors based on amorphous black phosphorus ultrathin films by pulsed laser deposition. Adv. Mater. 27, 3748-3754 (2015).

21. Kim, J. M. et al. Observation of tunable band gap and anisotropic Dirac semimetal state in black phosphorus. Science 349, 723-726 (2015)

22. Wang, X. M. et al. Highly anisotropic and robust excitons in monolayer black phosphorus. Nat. Nanotechnol. 10, 517-521 (2015).

23. Fei, R. X. \& Yang, L. Strain-engineering the anisotropic electrical conductance of few-layer black phosphorus. Nano Lett. 14, 2884-2889 (2014).

24. Schuster, R. et al. Anisotropic particle-hole excitations in black phosphorus. Phys. Rev. Lett. 115, 026404 (2015)

25. Qiao, J. S. et al. High-mobility transport anisotropy and linear dichroism in few-layer black phosphorus. Nat. Commun. 5, 4475 (2014).

26. Li, W. F. et al. Ultrafast and directional diffusion of lithium in phosphorene for high-performance lithium-ion battery. Nano Lett. 15, 1691-1697 (2015).

27. Li, Q. F. et al. Theoretical prediction of anode materials in Li-ion batteries on layered black and blue phosphorus. J. Phys. Chem. C 119, 8662-8670 (2015).

28. Zhao, S. J., Kang, W. \& Xue, J. M. The potential application of phosphorene as an anode material in Li-ion batteries. J. Mater. Chem. A 2, 19046-19052 (2014).

29. Guo, G. C. et al. Pristine and defect-containing phosphorene as promising anode materials for rechargeable Li batteries. J. Mater. Chem. A 3, 11246-11252 (2015).

30. Park, C. M. \& Sohn, H. J. Black phosphorus and its composite for lithium rechargeable batteries. Adv. Mater. 19, 2465-2468 (2007).

31. Sun, L. Q. et al. Electrochemical activity of black phosphorus as an anode material for lithium-ion batteries. $J$. Phys. Chem. C 116, 14772-14779 (2012).

32. Padilha, J. E. et al. Van der Waals heterostructure of phosphorene and graphene: tuning the Schottky barrier and doping by electrostatic gating. Phys. Rev. Lett. 114, 066803 (2015). 
33. Guo, H. Y. et al. Phosphorene nanoribbons, phosphorus nanotubes, and van der waals multilayers. J. Phys. Chem. C 118, 14051-14059 (2014).

34. Castellanos-Gomez, A. et al. Isolation and characterization of few-layer black phosphorus. 2D Mater. 1, 025001 (2014).

35. Das, S. et al. Tunable transport gap in phosphorene. Nano Lett. 14, 5733-5739 (2014).

36. Koening, S. P. et al. Electric field effect in ultrathin black phosphorus. Appl. Phys. Lett. 104, 103106 (2014).

37. Kou, L. Z., Chen, C. F. \& Smith, S. C. Phosphorene: fabrication, properties, and applications. J. Phys. Chem. Lett. 6, 2794-2805 (2015).

38. Liu, Q. H. et al. Switching a normal insulator into a topological insulator via electric field with application to phosphorene. Nano Lett. 15, 1222-1228 (2015).

39. Srivastava, P. K. \& Ghosh, S. Eliminating defects from graphene monolayers during chemical exfoliation. Appl. Phys. Lett. 102, 043102 (2013).

40. Zhang, L. et al. Controlled synthesis of few-layered graphene sheets on a large scale using chemical exfoliation. Carbon 48, 2367-2371 (2010).

41. Feng, H. B., Wu, Y. M. \& Li, J. H. Direct exfoliation of graphite to graphene by a facile chemical approach. Small 10, 2233-2238 (2014).

42. Ambrosi, A., Sofer, Z. \& Pumera, M. $2 \mathrm{H} \rightarrow 1 \mathrm{~T}$ phase transition and hydrogen evolution activity of $\mathrm{MoS}_{2}, \mathrm{MoSe}_{2}$, $\mathrm{WS}_{2}$ and $\mathrm{Wse}_{2}$ strongly depends on the $\mathrm{MX}_{2}$ composition. Chem. Commun. 51, 8450-8453 (2015).

43. Coleman, J. N. et al. Two-dimensional nanosheets produced by liquid exfoliation of layered materials. Science 331, 568-571 (2011).

44. Xia, Z. Y. et al. The exfoliation of graphene in liquids by electrochemical, chemical, and sonication-assisted techniques: a nanoscale study. Adv. Funct. Mater. 23, 4684-4693 (2013).

45. Su, C. Y. et al. High-quality thin graphene films from fast electrochemical exfoliation. ASC Nano 3, 2332-2339 (2011).

46. Wei, D. et al. Graphene from electrochemical exfoliation and its direct applications in enhanced energy storage devices. Chem. Commun. 48, 1239-1241 (2012).

47. Kang, J. et al. Solvent exfoliation of electronic-grade, two-dimensonal black phosphorus. ACS Nano 9, 3596-3604 (2015).

48. Yasaei, P. et al. High-quality black phosphorus atomic layers by liquid-phase exfoliation. Adv. Mater. 27, 1887-1891 (2015).

49. Brent, J. R. et al. Production of few-layer phosphorene by liquid exfoliation of black phosphorus. Chem. Commun. 50, 13338-13341 (2014).

50. Woomer, A. H. et al. Phosphorene: synthesis, scale-up, and quantitative optical spectroscopy. ACS Nano DOI: 10.1021/acsnano.5b02599 (2015).

51. Hanlon, D. et al. Liquid exfoliation of solvent-stabilised black phosphorus: applications beyond electronic. arXiv: 1501.01881 (2015).

52. Paton, K. R. et al. Scalable production of large quantities of defect-free few-layer grapheme by shear exfoliation in liquids. Nat. Mater. 13, 624-630 (2014).

53. Nicolosi, V. et al. Liquid exfoliation of layered materials. Science 340, 1420 (2013).

54. Tour, J. M. Scaling up exfoliation. Nat. Mater. 13, 545-546 (2014).

55. Varrla, E. et al. Turbulence-assisted shear exfoliation of graphene using household detergent and a kitchen blender. Nanoscale 6, 11810-11819 (2014).

56. Williams, D. B. \& Carter, C. B. Transmission electron microscopy: a textbook for materials science. 2nd ed. 2009, New York: Springer

57. Zhang, S. et al. Extraordinary photoluminescence and strong temperature/angle-dependent Raman responses in few-layer phosphorene. ACS Nano 8, 9590-9596 (2014).

58. Wu, J. X. et al. Identifying the crystalline orientation of black phosphorus using angle-resolved polarized Raman spectroscopy. Angew. Chem. Int. Ed. 54, 2366-2369 (2015).

59. Ribeiro, H. B. et al. Unusual angular dependence of the Raman response in black phosphorus. ACS Nano 9, 4270-4276 (2015).

60. Lu, W. L. et al. Probing the anisotropic behaviors of black phosphorus by transmission electron microscopy, angular-dependent Raman spectra, and electronic transport measurements. Appl. Phys. Lett. 107, 021906 (2015).

61. Nemes-Incze, P. et al. Anomalies in thickness measurements of graphene and few layer graphite crystals by tapping mode atomic force microscopy. Carbon 46, 1435-1442 (2008).

62. Eiko, M. et al. Nanoprobe characterization of $\mathrm{MoS}_{2}$ nanosheets fabricated by Li-intercalation. Jpn. J. App. Phys. 54, 08LB07 (2015).

63. Utomo, A. T., Baker, M. \& Pacek, A. W. Flow pattern, periodicity and energy dissipation in a batch rotor-stator mixer. Chem. Eng. Res. Des. 86, 1397-1409 (2008).

64. Gollub, J. O. \& Swinney, H. L. Onset of turbulence in a rotating fluid. Phys. Rev. Lett. 35, 927-930 (1975).

65. Boxall, J. A. et al. Droplet size scaling of water-in-oil emulsions under turbulent flow. Langmuir 28, 104-110 
(2012).

66. Wengeler, R. \& Nirschl, H. Turbulent hydrodynamic stress induced dispersion and fragmentation of nanoscale agglomerates. J. Colloid Interf. Sci. 306, 262-273 (2007).

67. Wang, F. et al. Tracking lithium transport and electrochemical reactions in nanoparticles. Nat. Commun. 3, 1201 (2012).

68. Su, Q. M. et al. In situ transmission electron microscopy observation of the conversion mechanism of $\mathrm{Fe}_{2} \mathrm{O}_{3}$ /graphene anode during lithiation-delithiation processes. ACS Nano 7, 9115-9121 (2013).

69.

70.

71.

72.

73.

74.

75.

76.

57. Bridgeman, P. W. Two new modifications of phosphorus. J. Am. Chem. Soc. 36, 1344-1363 (1914).

\section{Acknowledgements}

This work was supported by the National Basic Research Program of China (973 Program, Grant No. 2015CB352106), the National Natural Science Foundation of China (NSFC, Grant Nos. 51372039, 61574034, 51202028), the Jiangsu Province Science and Technology Support Program (Grant No. BK20141118), the Fundamental Research Funds for the Central Universities (Grant Nos. 2242013R30004), China Postdoctoral Science Foundation Funded Project (Grant No. 2014M550259, 2015T80480). The work at Brookhaven National Lab is supported by U.S. DOE-BES under Contract number DE-AC02-98CH10886. 


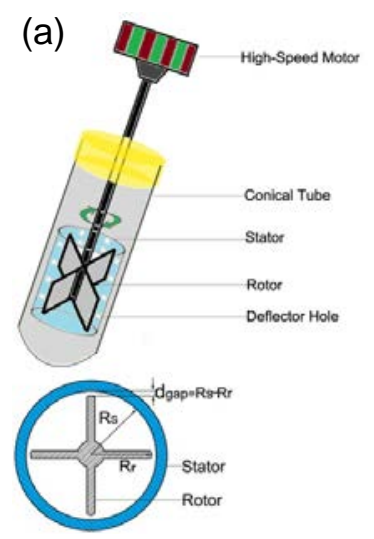

(b)

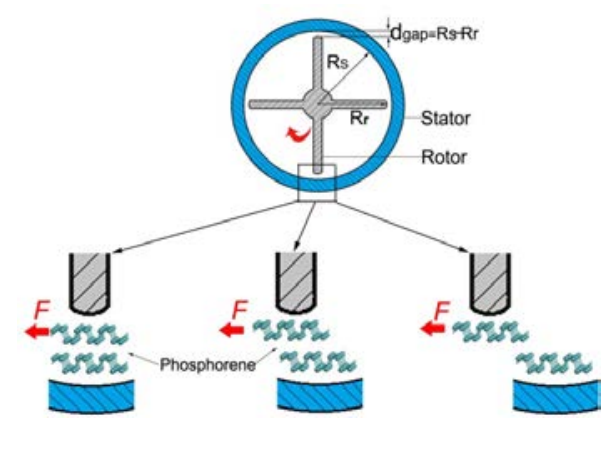

(c)

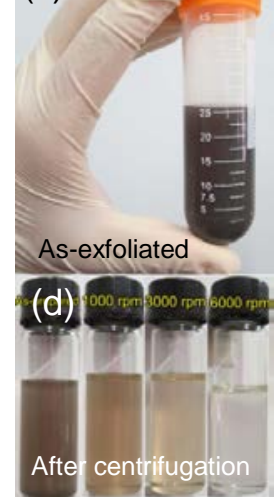

Figure 1 (a) Schematic of shear exfoliation setup for exfoliating bulk BP. (b) Schematic representing the shear-exfoliation process in the laminar flow regime. (c) Photograph of as-exfoliated phosphorene nanoflakes dispersed in NMP before centrifugation. (d) Photograph of phosphorene nanoflakes dispersed in NMP after 1000 rpm, 3000 rpm and 6000 rpm centrifugation, respectively. 

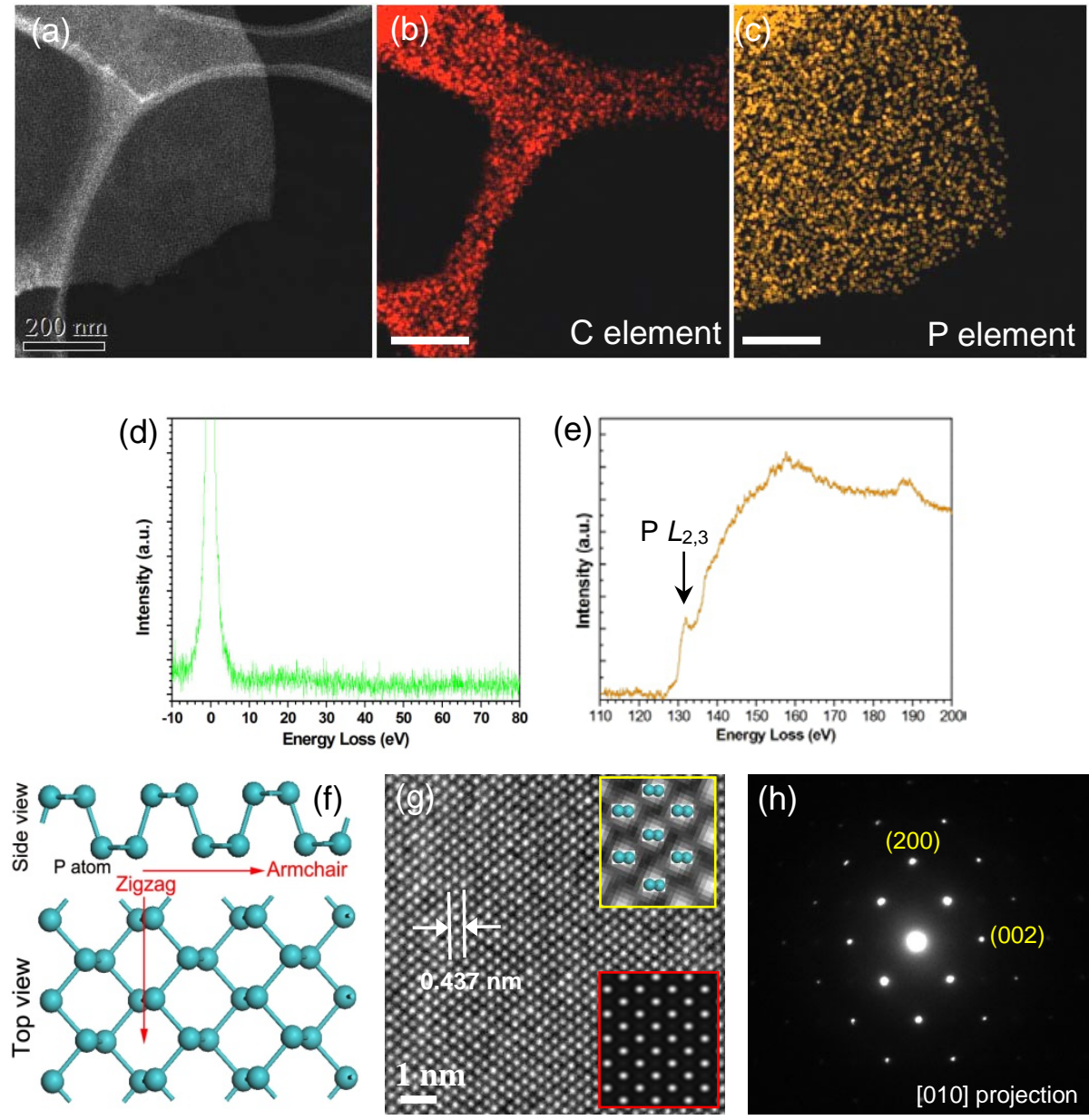

Figure 2 HAADF-STEM, EDS mapping, SAED, EELS characterization of phosphorene nanoflakes prepared by shear exfoliation and centrifugation at a rotation of $6000 \mathrm{rpm}$. (a) A typical STEM-HAADF image of an ultrathin phosphorene nanoflake on a lacy carbon support (scale bar is $200 \mathrm{~nm}$ ), revealing its uniformity in thickness. (b, c) EDS mapping of C and P elements corresponding to (a). Comparison between STEM-HAADF image and element mapping images indicates the high-purity of the phosphorene nanoflake after thoroughly filtrating and rinsing the shear-exfoliated products, no obvious $\mathrm{C}$ element from residual organic solvent is found on the surface of the nanoflake. (d) and (e) are low-loss and core-loss EELS of the phosphorene nanoflake. The characteristic core-loss peak at $133 \mathrm{eV}$ corresponding to the $\mathrm{P} L_{2,3}$ edge is observed clearly. Absence of the signature $\mathrm{P}_{x} \mathrm{O}_{y}$ peak loss confirms the pure phosphorus structure all over the nanoflake. The invisible plasmon peak in low-loss EELS (d) further proves that the nanoflake is extremely thin. (f) Schematic showing the atomic structure of phosphorene along side view and top view. (g) High-resolution TEM (HRTEM) image and (h) corresponding SAED pattern of the monolayer phosphorene corresponding to (a). The projection direction is along [010]. This HRTEM analysis provides strong evidence that high-quality monolayer phosphorene with highly crystalline nature can be produced by shear exfoliation. A filtered section of the HRTEM image (upper right inset in (g)) and TEM image simulation of monolayer phosphorene (lower right inset in (g)) are added for comparison. 

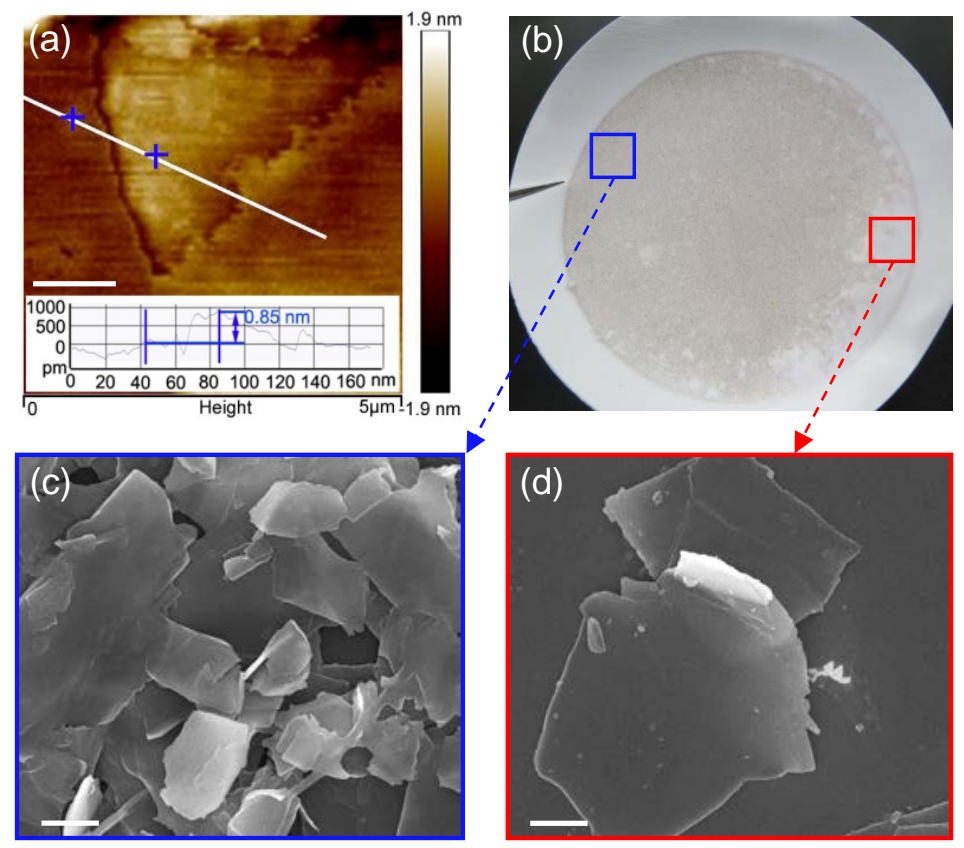

Figure 3 AFM and SEM characterization of shear-exfoliated phosphorene nanoflakes. (a) AFM height measurement of a monolayer phosphorene nanoflake on a $200 \mathrm{~nm} \mathrm{SiO}$-coated Si substrate. The nanoflake was isolated by centrifugating the as-exfoliated dispersions at a rotation speed of $6000 \mathrm{rpm}$. The height profile (inset) corresponds to the drawn white line. The stage height of around $0.85 \mathrm{~nm}$ proves the existence of a monolayer phosphorene nanoflake. Scale bar is $200 \mathrm{~nm}$. (b) Photograph and (c, d) SEM images of of as-filtrated film of phosphorene nanofalkes on polytetrafluoroethylene (PTFE) membrane, showing different size and dimensionality (scale bar is $2 \mu \mathrm{m}$ ). The nanoflakes were isolated by centrifugating at a low rotation speed of $3000 \mathrm{rpm}$. 

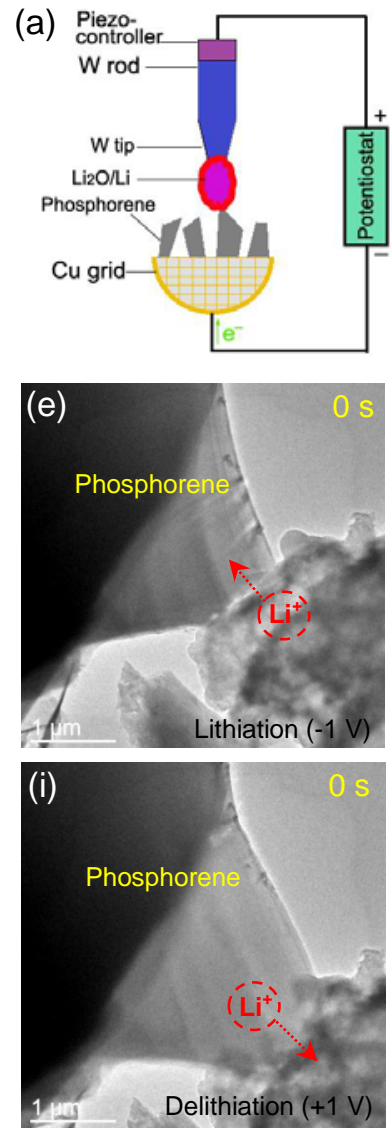

(b)
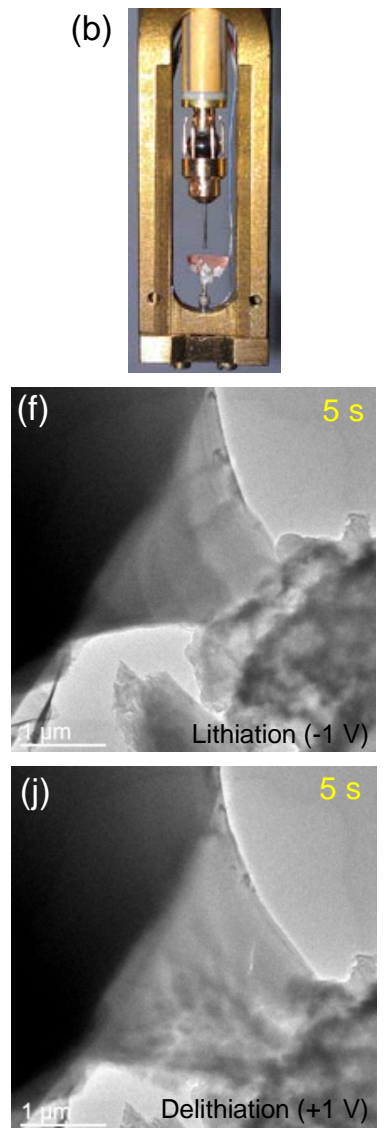
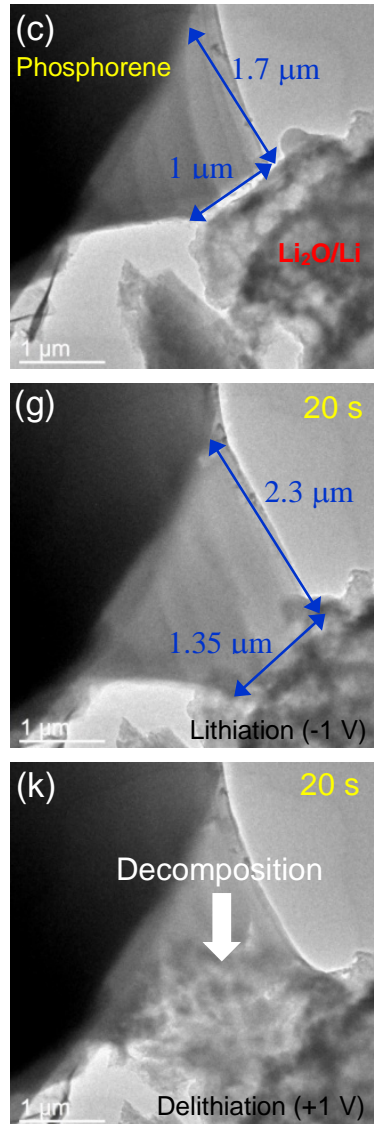
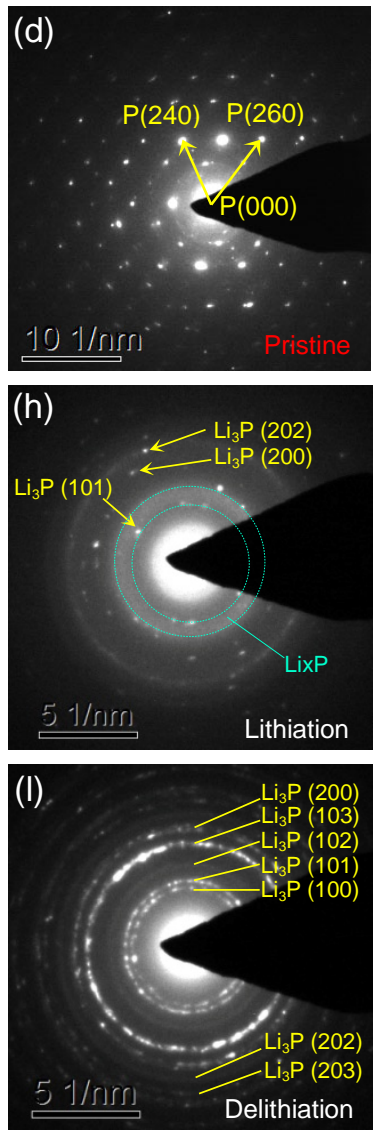

Figure 4 In situ TEM electrochemical lithiation/delithiation experiments of a slightly thick phosphorene nanoflake prepared by shear-exfoliation in NMP and centrifugation at a rotation speed of $1000 \mathrm{rpm}$. (a) Schematic and (b) Photograph of in situ lithiation experiment setup of TEM-STM sample holder. (c) TEM image of the nano-LIB constructed with an individual phosphorene nanoflake inside the TEM. (d) SAED pattern of the pristine phosphorene nanoflake, showing BP single-crystalline orthorhombic structure before lithiation. (e-g) SAED pattern of the lithiated phosphorene nanoflake, revealing the formation of $\mathrm{Li}_{3} \mathrm{P}$ after lithiation. Time-resolved TEM images from video frames show morphological evolutions of the phosphorene nanoflakes during the electrochemical lithiation process, and a lateral expansion of around 30\% was observed for the lithiated phosphorene nanoflakes. (g) (i-k) Time-resolved TEM images from video frames show morphological evolutions of the phosphorene nanoflake during the electrochemical delithiation process. (l) SAED pattern of the delithiated phosphorene nanoflake, revealing $\mathrm{Li}_{3} \mathrm{P}$ residual after delithiation. 

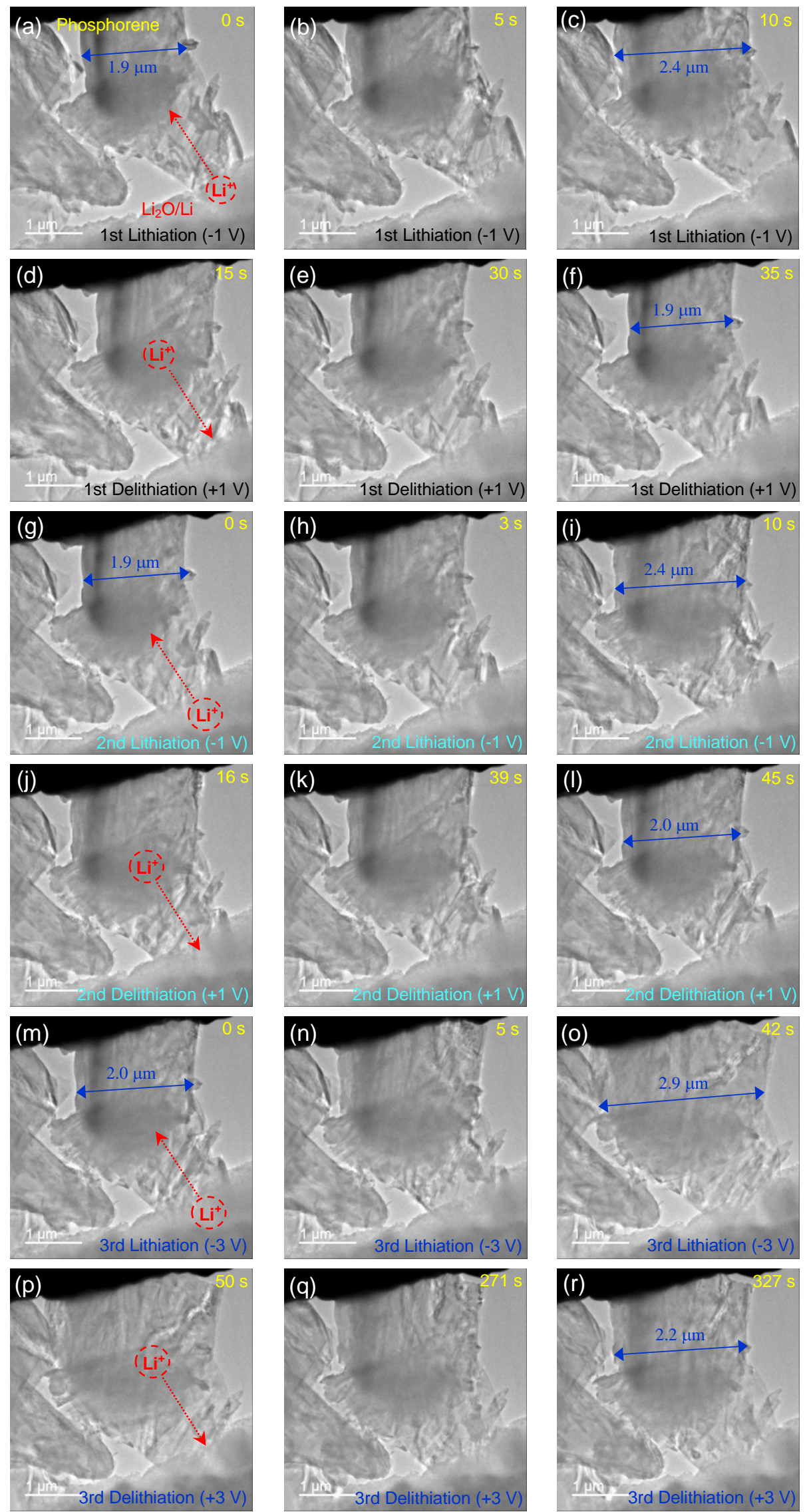

Figure 5 Multi-cycle in situ TEM electrochemical lithiation/delithiation experiments of an individual few-layer phosphorene nanoflake prepared by shear-exfoliation in NMP and centrifugation at a rotation speed of $6000 \mathrm{rpm}$. No decomposition phenomena took place during the delithiation, proving the superior structural stability compared with the thick phosphorene nanoflakes. (a-f) Time-resolved TEM images of the first lithiation/delithiation cycle, showing superior morphological restorability and structural stability. (g-l) Time-resolved TEM images of the second lithiation/delithiation cycle, maintaining the same morphological restorability and structural stability as the first lithiation/delithiation cycle. In both the lithiation/delithiation two cycles, the same potential of -1 V/1 V was applied. (m-r) Time-resolved TEM images of the third lithiation/delithiation cycle with a larger applied potential of $-3 \mathrm{~V} / 3 \mathrm{~V}$. Although a larger lateral expansion rate took place, the few-layer nanoflake still kept the superior morphological restorability and structural stability after the delithiation. 